Europhysics Letters

PREPRINT

\title{
The sediment of mixtures of charged colloids: segregation and inhomogeneous electric fields
}

\author{
Jos ZWANIKKEn and RENÉ van RoIJ \\ Institute for Theoretical Physics, Utrecht University, Leuvenlaan 4, 3584 CE Utrecht, \\ The Netherlands \\ PACS. 82.70.Dd - Colloids. \\ PACS. 05.20.Jj - Statistical mechanics of classical fluids.
}

\begin{abstract}
We theoretically study sedimentation-diffusion equilibrium of dilute binary, ternary, and polydisperse mixtures of colloidal particles with different buoyant masses and/or charges. We focus on the low-salt regime, where the entropy of the screening ions drives spontaneous charge separation and the formation of an inhomogeneous macroscopic electric field. The resulting electric force lifts the colloids against gravity, yielding highly nonbarometric and even nonmonotonic colloidal density profiles. The most profound effect is the phenomenon of segregation into layers of colloids with equal mass-per-charge, including the possibility that heavy colloidal species float onto lighter ones.
\end{abstract}

Introduction. - Sedimentation in a suspension of colloidal particles is of profound fundamental importance, and has been studied in detail for a long time. For instance, in 1910 Perrin determined the Boltzmann constant $k_{B}$ (and from this Avogadro's number) by comparing the measured equilibrium density profile of a dilute suspension with the theoretically predicted barometric law [1], and more recently the full hard-sphere equation of state was determined accurately from a single measurement of the sedimentation profile of a dense suspension of colloidal hard spheres $[2,3]$. In rather dilute suspensions of charged colloids, however, strong deviations from the barometric distribution have recently been theoretically predicted [4-9], experimentally observed [10-13], and simulated [14], at least in the regime of extremely low salinity. The most striking phenomenon is that the distribution of colloids extends to much higher altitudes than is to be expected on the basis of their buoyant mass. The force that lifts the colloids against gravity is provided by an electric field that is induced by spontaneous charge separation over macroscopic distances. The corresponding cost of electrostatic and gravitational energy is more than compensated by the gain of translational entropy of the salt ions, which are distributed much more homogeneously than when they would have had to follow the colloids to the bottom part of the suspension. The same entropy-induced lifting force was recently found to be responsible for a so-called Brazil-nut effect in binary mixtures of charged colloidal particles under low-salt conditions: the heavy particles can reside at higher altitudes than the lighter ones [15]. In this Letter we extend the theoretical study of sedimentation of charged colloids to binary, ternary, and polydisperse mixtures, for which

(C) EDP Sciences 
we calculate colloidal density profiles and the entropy-induced electric field selfconsistently within a Poisson-Boltzmann like density functional theory.

Theory. - We consider an $n$-component suspension of negatively charged colloidal spheres in a salt solution in the Earth's gravity field. We label the colloidal species by $i=1,2, \cdots, n$, and denote the electric charge, diameter, and buoyant mass for species $i$ by $-Z_{i} e, \sigma_{i}$, and $m_{i}$, respectively, where $e$ is the proton charge. We imagine the suspension to be in thermal and osmotic contact with a reservoir that contains monovalent ions at a concentration $2 \rho_{s}$ and a solvent with dielectric constant $\epsilon$ at temperature $T$. The ions are assumed to be massless point particles with charge $\pm e$, and the solvent mass density is taken into account by considering the buoyant instead of the actual colloidal masses according to Archimedes. The volume of the suspension is $V=A H$, with $A$ the (macroscopic) horizontal area and $H$ the vertical height of the solvent meniscus above the bottom of the system. The gravitational acceleration $g$ is in the negative vertical direction. We are interested in the equilibrium colloidal density profiles $\rho_{i}(x)$ as a function of the altitude $x$ above the bottom at $x=0$ and below the meniscus at $x=H$. The ion density profiles are denoted by $\rho_{ \pm}(x)$. In order to calculate these profiles we employ the framework of density functional theory [16], where the equilibrium density profiles follow from the minimisation of a grand potential functional $\Omega\left[\left\{\rho_{i}\right\}, \rho_{ \pm}\right]$with respect to all the profiles. Here we employ the mean-field free energy functional that consists of entropic, gravitational, and electrostatic contributions,

$$
\begin{aligned}
\frac{\Omega}{k_{B} T A} & =\sum_{\alpha= \pm} \int_{0}^{H} \mathrm{~d} x \rho_{\alpha}(x)\left(\ln \frac{\rho_{\alpha}(x)}{\rho_{s}}-1\right)+\sum_{i=1}^{n} \int_{0}^{H} \mathrm{~d} x \rho_{i}(x)\left(\ln \frac{\rho_{i}(x)}{a_{i}}-1\right) \\
& +\sum_{i=1}^{n} \int_{0}^{H} \mathrm{~d} x \frac{x}{L_{i}} \rho_{i}(x)-\frac{2 \pi \lambda_{B}}{2} \int_{0}^{H} \mathrm{~d} x \int_{0}^{H} \mathrm{~d} x^{\prime}\left|x-x^{\prime}\right| Q(x) Q\left(x^{\prime}\right),
\end{aligned}
$$

where we introduced the fugacity (or activity) $a_{i}$ and the gravitational length $L_{i}=k_{B} T / m_{i} g$ of species $i$, the Bjerrum length $\lambda_{B}=e^{2} / \epsilon k_{B} T$, and the local charge density $Q(x)=\rho_{+}(x)-$ $\rho_{-}(x)-\sum_{i=1}^{n} Z_{i} \rho_{i}(x)$. The electrostatic term follows from an in-plane integration of the threedimensional Coulomb law, $2 \pi \int_{0}^{\infty} \mathrm{d} R R / \sqrt{\left|x-x^{\prime}\right|^{2}+R^{2}}=-2 \pi\left|x-x^{\prime}\right|$ with $R$ the in-plane distance, where we ignored an irrelevant integration constant. The functional (1) only couples the total charge density at different heights in a mean-field fashion, i.e. electric double layers and many other correlation effect are not taken into account. In fact one easily checks that the thermodynamics of the system reduces in the absence of gravity to an $(n+2)$-component idealgas mixture, since then $Q(x) \equiv 0$ because of translational invariance and charge neutrality. In the presence of gravity, however, interesting structures already appear at this relatively low level of sophistication. The Euler-Lagrange equations $\delta \Omega / \delta \rho_{\alpha}(x)=0$ and $\delta \Omega / \delta \rho_{i}(x)=0$ that must be satisfied by the the equilibrium density profiles can be cast in the form

$$
\begin{aligned}
\rho_{ \pm}(x) & =\rho_{s} \exp [\mp \phi(x)] ; \\
\rho_{i}(x) & =a_{i} \exp \left[-x / L_{i}+Z_{i} \phi(x)\right] ; \quad(i=1,2, \cdots, n)
\end{aligned}
$$

where $\phi(x)=-2 \pi \lambda_{B} \int_{0}^{H} \mathrm{~d} x^{\prime} Q\left(x^{\prime}\right)\left|x-x^{\prime}\right|$ is the dimensionless electrostatic potential gauged such that it is zero in the reservoir where $Q(x) \equiv 0$. It turns out to be convenient to rewrite it in differential form as the Poisson-Boltzmann equation

$$
\frac{\mathrm{d}^{2} \phi(x)}{\mathrm{d} x^{2}}=-4 \pi \lambda_{B} Q(x)=\kappa^{2} \sinh \phi(x)+4 \pi \lambda_{B} \sum_{i=1}^{n} Z_{i} \rho_{i}(x) .
$$


Here we used Eq.(2), and we defined the reservoir screening constant $\kappa^{2}=8 \pi \lambda_{B} \rho_{s}$. Together with the boundary conditions $\phi^{\prime}(0)=\phi^{\prime}(H)=0$ (where a prime denotes a derivative w.r.t. $x$ ) the Eqs. (3) and (4) form a closed set of $n+1$ equations that can in principle be solved to yield $\rho_{i}(x)$ and $\phi(x)$ for a given thermodynamic state determined by the reservoir characteristics $\kappa$ and $\lambda_{B}$, and by the colloidal fugacities $a_{i}$, gravitational lengths $L_{i}$, and charges $Z_{i}$. In an experimental situation, however, the total packing fraction $\bar{\eta}_{i}=(\pi / 6) N_{i} \sigma_{i}^{3} / V$ of species $i$ is usually fixed instead of the fugacity $a_{i}$, with $N_{i}$ the number of colloids of species $i$. This conversion can easily be accomplished here by regarding the fugacities $a_{i}$ as normalization constants that take values such that $\bar{\eta}_{i}=(1 / H) \int_{0}^{H} \mathrm{~d} x \eta_{i}(x)$, with $\eta_{i}(x)=(\pi / 6) \rho_{i}(x) \sigma_{i}^{3}$ the local packing fraction of species $i$. Note that the hard-core diameters $\sigma_{i}$ do not appear at all in the Euler-Lagrange equations, since the functional (1) ignores the hard-core part of the (direct) correlations; we use for all colloidal species that $\sigma_{i}=\sigma=150 \mathrm{~nm}$ only to be able to convert densities to physically reasonable packing fractions. Once the profiles $\phi(x)$ and $\rho_{i}(x)$ are known, then $\rho_{ \pm}(x)$ follows from Eq.(2), and from this the total charge density $Q(x)$. The magnitude of the electric field is given by $E(x)=k_{B} T \phi^{\prime}(x) / e$, where the prime denotes a derivative w.r.t. the height $x$.

Unfortunately one cannot solve Eqs.(3) and (4) analytically. However, its one-dimensional character allows for a rather straightforward numerical solution on a grid $\left\{x_{k}\right\}$ of heights, although some care must be taken in dealing with the widely different length scales $H$ (say of the order of centimeters) and $\kappa^{-1}$ (at most of the order of microns) in realistic cases. In all our calculations we use a non-equidistant grid that we adapt to the situation at hand, making sure that it is fine enough to exclude numerical artifacts. Typically we use 600 grid points for a system with a meniscus at $H=20 \mathrm{~cm}$. Our iterative scheme to solve Eqs.(3) and (4) on the grid takes typically a few seconds on a desktop PC for $n=1$, and a minute for $n=21$.

Before discussing our numerical results, we wish to point out that the present theory reduces, for $n=1$, to the treatment of Ref. [8]. For instance, the three regimes for the colloidal density profile (barometric, linear, and exponential with a large decay length) follow within the assumption of local charge neutrality $Q(x)=0$, i.e. $-\sinh \phi(x)=Z_{1} \rho_{1}(x) / 2 \rho_{s} \equiv y_{1}(x)$. In the linear regime, where $1 / Z_{1}<y_{1}(x)<1$, the potential is then from Eq.(4) linear in $x$, with a slope $\phi^{\prime}(x)=1 / Z_{1} L_{1}$ that is proportional to the electric field that lifts the colloids against gravity [8]. This one-component result already hints at an important complication for mixtures $(n \geq 2)$ : since generally $Z_{i} L_{i} \neq Z_{j} L_{j}$ a single electric field strength cannot lift all the colloidal species simultaneously. On this basis one could therefore already expect segregation in mixtures, such that colloids with the same value $Z_{i} L_{i}$ (i.e. the same charge-per-mass) are found at the same height. This is indeed what our numerical results will show below.

Segregation in binary and ternary mixtures. - We start our numerical investigation with a class of binary mixtures $(n=2)$ of equal-sized light $(i=1)$ and heavy $(i=2)$ colloidal particles of various charge ratios. We choose the system parameters identical to those of the Monte Carlo simulations of figure 3 of Ref. [15]: $\sigma=150 \mathrm{~nm}, H=1000 \sigma, \lambda_{B}=\sigma / 128$, $L_{1}=\frac{3}{2} L_{2}=10 \sigma, \bar{\eta}_{1}=\bar{\eta}_{2}=(\pi / 6) \times 10^{-4}, Z_{1}=15$, and $Z_{2}$ varies between 15 and 45 . The simulated system does not contain added salt but only counterions, which we can represent within our theory by the extremely low reservoir salt concentration $\rho_{s}=1 \mathrm{nM}$, which we checked to be low enough to be in the zero-added-salt limit as regards the colloidal profiles. In Fig.1(a) we show, for $Z_{2}=45$, the density profiles of the two colloidal species, as well as that of the counterions (and the coions) in the inset. We observe profound colloidal segregation into two layers, with the heavy species floating on top of the lighter ones. The counterions are seen to be distributed throughout the whole volume, i.e. much more homogeneously than when all colloids would have been barometrically distributed in a thin layer of thickness $L_{i}$ 
just above the bottom (since in that case the net ion charge would have been located in that same thin layer). The resulting gain of ion entropy is the driving force for the formation of the electric field that pushes the heavy (highly charged) colloids to high altitudes against gravity $[8,14]$. In Fig.1(b) we show the mean height $h_{i}$ of species $i$, defined as

$$
h_{i}=\frac{\int_{0}^{H} \mathrm{~d} x x \rho_{i}(x)}{\int_{0}^{H} \mathrm{~d} x \rho_{i}(x)},
$$

as a function of $Z_{2}$. We replot the Monte Carlo simulation results of figure 3 of Ref. [15] (symbols), together with the predictions for $h_{i}$ that follow from the present theory (continuous curves). Given that there is not a single fit parameter involved, the agreement is remarkable, certainly when compared with the theoretical analysis on the basis exponentially decaying density profiles as in Ref. [15]. Fig.1 shows that the heavy particles are on top of the lighter ones, $h_{2}>h_{1}$, provided $Z_{2} / Z_{1} \simeq 1.6$, a phenomenon that was termed the "colloidal Brazil nut effect" in Ref. [15]. For barometric profiles one would find that $h_{i}=L_{i}$, but we see in all cases that $h_{i} \gg L_{i}$ due to the lift effect of the induced electric field. The good agreement between theory and simulation in Fig.1(b) also indicates that hard-core effects (which are taken into account in the simulations but not in the theory) are not so relevant, at least not in the parameter regime studied here.
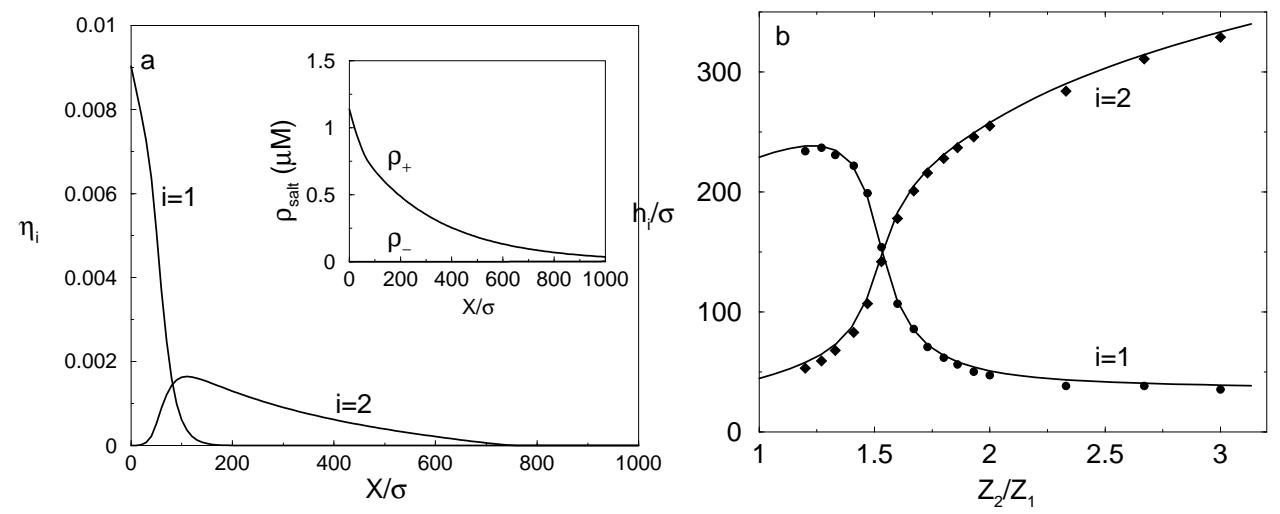

Figure 1 - (a) Colloidal density profiles and counterion distribution (inset) for a deionised binary mixture of equal-sized light $(i=1)$ and heavy $(i=2)$ colloidal spheres (diameter $\sigma=150 \mathrm{~nm}$ ), with meniscus height $H=1000 \sigma$, gravitational lengths $L_{1}=10 \sigma$ and $L_{2}=20 \sigma / 3$, average packing fractions $\bar{\eta}_{1}=\bar{\eta}_{2}=(\pi / 6) \times 10^{-4}$, colloidal charges $Z_{1}=15$ and $Z_{2}=45$, and Bjerrum length $\lambda_{B}=\sigma / 128$. (b) Mean height $h_{i}$ of the two species for the same system as in (a) except now for a range of $Z_{2}$, showing a density inversion $\left(h_{2}>h_{1}\right.$, the heavy particles floating on top of light ones) for $Z_{2} / Z_{1}>1.6$. The curves are predictions of the present theory, the symbols are simulation data of Ref. [15].

The next system we study is a ternary system $(n=3)$ in a solvent characterised by $\lambda_{B}=2.3 \mathrm{~nm}$ (ethanol at room temperature) with a reservoir salt concentration $\rho_{s}=10 \mu \mathrm{M}$ and meniscus height $H=20 \mathrm{~cm}$. The colloidal charges and gravitational lengths are $Z_{i}=$ $(1000,250,125)$ and $L_{i}=(1,2,1) \mathrm{mm}$, respectively, and the system is equimolar with $\bar{\eta}_{i}=$ 0.005 for all three species (recall that all species have the same diameter $\sigma=150 \mathrm{~nm}$ ). Fig. 2 shows the density profiles as predicted by the present theory in (a), as well as the electric field in (b). We find almost perfect segregation into three layers, such that the mean heights satisfy 
$h_{3}<h_{2}<h_{1}$. Note that this ordering coincides with the ordering $Z_{3} L_{3}<Z_{2} L_{2}<Z_{1} L_{1}$, and not with the corresponding ordering of $L_{i}$ which one would expect on the basis of a barometric distribution. In other words, this system segregates according to mass-per-charge instead of the more usual ordering according to mass: the colloids with the largest mass-per-charge are found at the bottom. In the present case this implies that the lightest colloids (species 2) are found in a layer in between the equally heavy species 1 and 3. On the basis of the onecomponent theory of Ref. [8] one would expect a linearly decaying density profile of species $i$ in the layer where species $i$ is dominant, as well as a linear electrostatic potential. Denoting the derivative w.r.t. $x$ by a prime, the one-component theory predicts $\eta_{i}^{\prime}(x)=\pi \rho_{s} \sigma^{3} / 3 Z_{i}^{2} L_{i}$ and $\phi^{\prime}(x)=1 / Z_{i} L_{i}$ in the layer with species $i$, corresponding to an electric field strength $m_{i} g / Z_{i} e$ in this layer. These values for the density gradients and the electric field are indicated in Fig.2(a) and (b), respectively, and are in good agreement with the numerical results. The result for the electric field can also be easily obtained analytically for a mixture provided one assumes that segregation takes place, such that $\rho_{i}(x)$ takes a maximum at some height $x^{*}$ in the layer of species $i$. From $\rho_{i}^{\prime}\left(x^{*}\right)=0$ one obtains from Eq.(3) that $\phi^{\prime}\left(x^{*}\right)=1 / Z_{i} L_{i}$, in agreement with the numerical results. The inset of Fig.2(b) shows the ratio of the total charge density $Q(x)$ and the ion charge density $2 \rho_{s} \sinh \phi(x)$, which is such that $\left|Q(x) / 2 \rho_{s} \sinh \phi(x)\right| \ll 1$ for all $x$ except close to $x=0$ and $x=H$ where it is $\sim 1$. This indicates that the system, with its inhomogeneous electric field, is yet essentially locally charge neutral (but not exactly, and not at all at the boundaries), suggesting that a description on the basis of hydrostatic equilibrium within a local density approximation should be rather accurate [17].
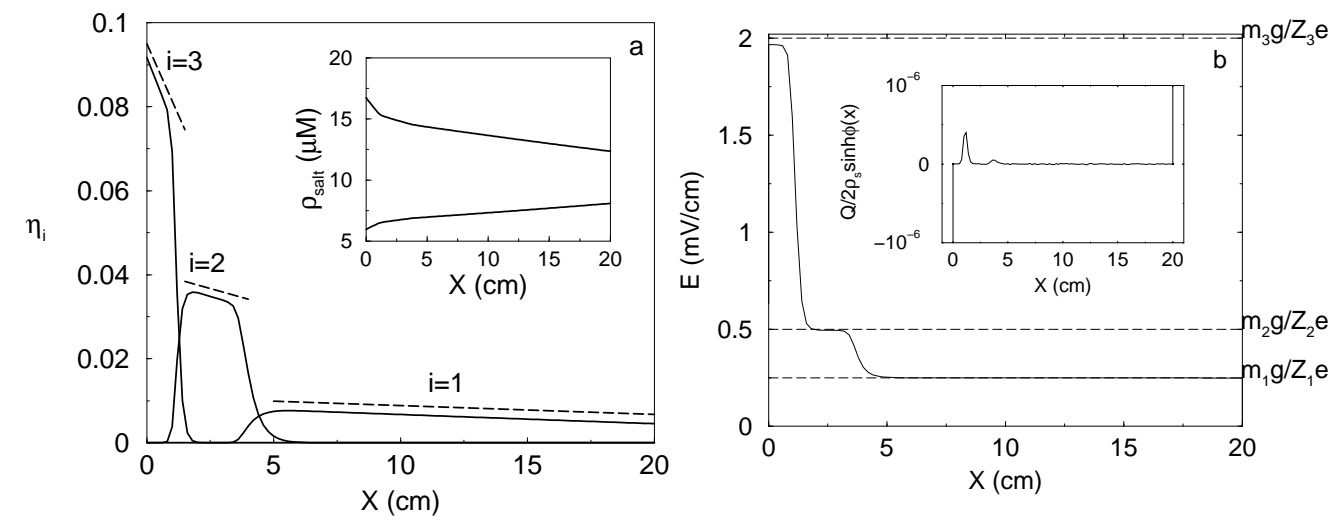

Figure 2 - Density profiles (a) and the electric field profile (b) of an equimolar ternary mixture of equally-sized colloids with charges $Z_{i}=(1000,250,125)$ and gravitational lengths $L_{i}=(1,2,1) \mathrm{mm}$ with total packing fractions $\bar{\eta}_{i}=0.005$ in a $20 \mathrm{~cm}$ ethanol suspension at room temperature, with monovalent ionic strength $\rho_{s}=10 \mu \mathrm{M}$ in the reservoir. Almost complete segregation takes place into well-defined layers of pure components, with a slope $\eta_{i}^{\prime}(x)=\pi \rho_{s} \sigma^{3} / 3 Z_{i}^{2} L_{i}$ and a constant electric field $m_{i} g / e Z_{i}$ in the layer with component $i$, as denoted by the dashed lines in (a) and (b), respectively. The inset in (b) shows a dimensionless measure for local charge neutrality (see main text).

Polydisperse Mixtures. - We now extend our study to polydisperse mixtures, where one could expect segregation into many layers on the basis of the results for two and three components. We mimic the polydispersity by considering a system of $n=21$ components, with $Z_{i}$ distributed as a Gaussian with average of 250 and a standard deviation of 50 . This distribution is shown in the inset of Fig. 3(a), where the vertical axis $\left(\bar{\eta}_{i}\right)$ is proportional to 
the relative frequency of species $i$ in the sample. We consider two distributions for $L_{i}$ : (A) $L_{i}=2 \mathrm{~mm}$ for all species, and (B) $L_{i}=2 \times\left(250 / Z_{i}\right)^{3 / 2} \mathrm{~mm}$. Case B mimics the situation for spheres of different size but the same mass density and surface charge density, such that $Z_{i}$ is proportional to the surface area and $L_{i}$ to the inverse volume of species $i$, i.e. $L_{i}^{2} Z_{i}^{3}$ is a constant independent if $i$. The density profiles, numerically obtained by solving the Eqs.(3i) and (44) for $H=20 \mathrm{~cm}, \lambda_{B}=2.3 \mathrm{~nm}, \rho_{s}=3 \mu \mathrm{M}$, and $\bar{\eta}_{t o t}=\sum_{i=1}^{21} \bar{\eta}_{i}=0.005$, are shown in Fig.3 for all 21 components. Case A shows profound lifting and layering, where the ordering is again determined by mass-per-charge as illustrated by the three dashed curves (for $Z_{i}=250,279$, and 299) showing that the colloids in the high-charge wing of the distribution reside at high altitudes. Fig.3(b) shows the density profiles for case B, which does exhibit lifting, but hardly any layering, and no density inversion at all. This is completely consistent with the picture that the ordering is determined by $Z_{i} L_{i}$, which for case $\mathrm{B}$ is such that $Z_{i} L_{i} / Z_{j} L_{j}=\sqrt{Z_{j} / Z_{i}}$, i.e. the highly-charged particle are expected at the bottom while the relative spread in $Z_{i} L_{i}$ is relatively small compared to case A, where $Z_{i} L_{i} / Z_{j} L_{j}=Z_{i} / Z_{j}$. The inset of (b) shows the total packing fraction profiles $\eta_{t o t}(x)=\sum_{i=1}^{21} \eta_{i}(x)$ of both case $\mathrm{A}$ and $\mathrm{B}$ together with the one-component profile $(n=1)$ with $Z_{1}=250$ and $L_{1}=2 \mathrm{~mm}$ at $\bar{\eta}_{1}=0.005$. Perhaps surprisingly there is hardly any distinction between the pure system and case B, whereas there is a small difference with case A. These profiles show that the main distinction between these polydisperse systems and the underlying one-component one concerns the layering phenomenon (provided $Z_{i} L_{i}$ varies sufficiently for all the species), and not the total distribution of the colloids. Perhaps this fractionation effect could be exploited experimentally to purify a polydisperse mixture.
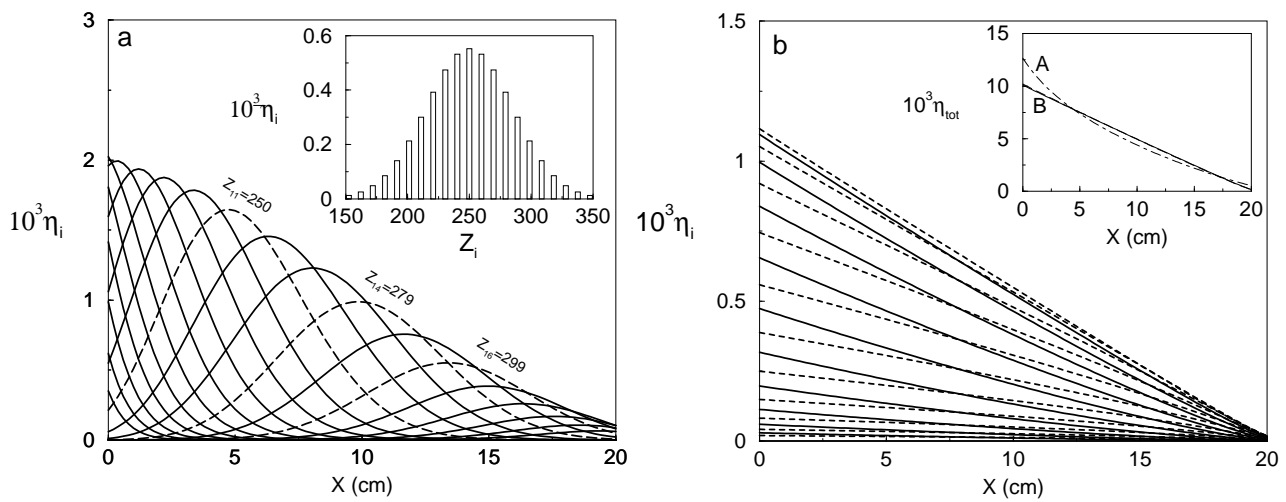

Figure 3 - Density profiles of a 21-component colloidal suspension with a Gaussian charge distribution as illustrated in the inset of (a), at total packing fraction 0.005 , reservoir salt concentration $\rho_{s}=3 \mu \mathrm{M}$, and Bjerrum length $\lambda_{B}=2.3 \mathrm{~nm}$. The gravitational lengths are as in case A (see main text) in (a), and case $\mathrm{B}$ in (b). In (b) the solid curves are for $Z_{i}>250$, and the dashed ones for $Z_{i} \leq 250$. The inset of (b) shows the total packing fraction profile for case A and B, together with that of the underlying one-component system (see main text).

Conclusions and discussion. - We have studied sedimentation equilibrium of $n$-component systems of charged colloidal particles at low salinity within a Poisson-Boltzmann-like density functional. The Euler-Lagrange equations that describe the minimisation of the functional are solved numerically on a one dimensional grid of heights for a number of system parameters. For $n=1$ the theory reduces to the one-component studies as presented in 
Ref. [8,14], and for $n=2$ we quantitatively reproduce the simulation results of Ref. [15], where density inversion was found such that the heavier colloids float on top of the lighter ones. This effect is caused by a self-consistent electric field that lifts the higher charged (heavy) particles to higher altitudes than the lower charged (lighter) colloids. We show that the layering of the colloids according to mass-per-charge can persist for ternary $(n=3)$ as well as for polydisperse (here $n=21$ ) mixtures. Given the good account that the present theory gives for simulations [14] and experiments [11-13] of one-component systems, and for the simulations of binary systems [15], it is tempting to argue that the theory is also (qualitatively) reliable for ternary or polydisperse systems, i.e. the predicted segregation and layering should be experimentally observable. One should bare in mind, however, that the present theory ignores the hard-core of the colloidal particles, and is therefore expected to break down at higher packing fractions. It also ignores effects due to charge renormalisation, which becomes relevant when $Z_{i} \lambda_{B} / \sigma_{i} \gg 1$. Work on extending the theory in these directions is in progress $[17,18]$.

All results presented in this paper were obtained with the zero-field boundary conditions $\phi^{\prime}(0)=\phi^{\prime}(H)=0$. We checked explicitly, however, that other boundary conditions that respect global charge neutrality, such as $\phi^{\prime}(0)=\phi^{\prime}(H)=e E_{e x t} / k_{B} T$ (describing a suspension in a homogeneous external electric field $E_{\text {ext }}$ ) or $\phi(0)=\phi(H)$ and $\phi^{\prime}(0)=\phi^{\prime}(H)$ (describing a short-circuited bottom and meniscus) give indistinguishable density profiles, except in two layers of thickness $\sim \kappa^{-1} \ll 10 \mu \mathrm{m}$ in the vicinity of the bottom and the meniscus. This insensitivity to the boundary conditions is not surprising in the light of the fact that the whole phenomenology in these systems is driven by the entropy of the microscopic ions, i.e. a bulk contribution to the grand potential that should dominate any boundary (surface) contribution.

Acknowledgements. - It is a pleasure to thank Maarten Biesheuvel for interesting discussions. This work is part of the research programme of the "Stichting voor Fundamenteel Onderzoek der Materie (FOM)", which is financially supported by the "Nederlandse organisatie voor Wetenschappelijk Onderzoek (NWO)".

References

[1] J. Perrin, (1913), Les Atomes (Paris: Alcan).

[2] R. Piazza, T. Bellini, V. Degiorgio, Phys. Rev. Lett. 71, 4267 (1993).

[3] M.A. Rutgers, J-Z Xue, W.B. Russel, and P.M. Chaikin, Phys. Rev. B 53, 5043 (1996).

[4] T. Biben and J.-P. Hansen, J. Phys.:Cond. Matt. 6, A345 (1994).

[5] J.-P. Simonin, J. Phys. Chem. 99, 1577 (1995).

[6] G. Téllez and T. Biben, Eur. Phys. J. E 2, 137 (2000).

[7] H. Löwen, J. Phys.: Cond. Matt. 10, L479 (1998).

[8] R. van Roij, J. Phys.: Condens. Matter 15, S3569 (2003).

[9] M. Biesheuvel, J. Phys.:Cond. Matt. 16, L499 (2004)

[10] A.P. Philipse and G.H. Koenderink, Adv. in Colloid and Interface Science 100-102, 613 (2003).

[11] M. Rasa and A.P. Philipse, Nature 429, 857 (2004).

[12] C.P. Royall, R. van Roij, and A. van Blaaderen, J.Phys.:Cond. Matt. 17, 2315 (2005).

[13] M. Rasa, B. Erné, B. Zoetekouw, R. van Roij, and A.P. Philipse, J.Phys.:Cond. Matt. 17, 2293 (2005).

[14] A.P. Hynninen, R. van Roij, and M. Dijkstra, Europhys. Lett. 65, 719 (2004).

[15] A. Esztermann and H. Löwen, Europhys. Lett, 68, 120 (2004).

[16] R. Evans, Adv. Phys. 28, 143 (1979).

[17] A. Torres and R. van Roij, to be published.

[18] M. Biesheuvel, private communications and preprint. 